\title{
Comment on: "Effect of sustained virologic response on the incidence of hepatocellular carcinoma in patients with HCV cirrhosis"
}

\author{
Authors \\ Fanpu Ji ${ }^{1}$ \\ Zhifang $\mathrm{Cai}^{2}$ \\ Hong Deng ${ }^{3}$ \\ ${ }^{1}$ Attending Physician, \\ Department of Infectious \\ Disease, The Second \\ Affiliated Hospital, College \\ of Medicine, Xian Jiaotong \\ University, China \\ ${ }^{2}$ Associate Professor \\ Department of Infectious \\ Disease, The Second \\ Affiliated Hospital, College \\ of Medicine, Xian Jiaotong \\ University, China \\ ${ }^{3}$ Professor, Department \\ of Infectious Disease, The \\ Second Affiliated Hospital, \\ College of Medicine, Xi'an \\ Jiaotong University, China
}

Submitted on: $02 / 15 / 2011$ Approved on: 02/15/2011

Correspondence to:

Fanpu Ji

157 Xi Wu Road, Xi'an

710004

Shaanxi Province, PR

China

jifanpu1979@163.com

Financial Support:

Health Research

Foundation of Shaanxi

Province, China, No.

2010H31

We declare no conflict of interest.

(C)2011 Elsevier Editora Ltda. All rights reserved.
Dear Editor,

We read with great interest the study by Cheinquer et al. ${ }^{1}$ in a recent issue of the Brazilian Journal of Infectious Diseases where they suggest that patients with cirrhosis due to hepatitis C virus (HCV) who achieved sustained virological response (SVR) had significantly lower incidence of hepatocellular carcinoma (HCC) when compared to those without treatment response. Also, interferon treatment after curative resection or ablation of HCC in HCV related cirrhotics prevents HCC recurrence and improves survival, especially for patients with SVR, had been reported in a recent meta-analysis. ${ }^{2}$

However, a large-scale randomized controlled trial (the HALT-C study) showed no significant difference in the incidence of HCC between patients on maintenance interferon therapy and those without. ${ }^{3}$ The reason for the discrepant results in studies needs further clarification, together with analysis of the difference in incidence rates of HCC among cirrhotic patients.

A prospective study was carried out to investigate the efficacy and safety of standard interferon with a low progressive dosage regimen in combination with ribavirin in patients with HCV-related decompensated cirrhosis in our center since 2008. Two patients with HCV-related decompensated cirrhosis developed HCC after successful antiviral therapy. Both of them had been treated with standard interferon $a-2 b$ ( $3 \mathrm{Mu}$, every other day) and ribavirin (800 $\mathrm{mg} / \mathrm{d}$ ) for 48 weeks. The first case (62-yearold, female, Child-Pugh score 8 and genotype 1b) developed a hepatocellular carcinoma at month 6 after achieving SVR. The second case (53-year-old, male, Child-Pugh score 9 and genotype $2 \mathrm{a}$ ) achieved early virologic response and end of treatment response, a single $2 \mathrm{~cm}$ tumor nodule was detected by enhanced CT liver scan three months after end of treatment.
Both patients underwent transcatheter arterial chemoembolization due to expansion and shortage of organs.

Although SVR is associated with long term good prognosis for patients with HCV infection, ${ }^{4,5}$ two out of 28 cirrhotic patients developed HCC compared to none of 146 patients without cirrhosis during follow-up for a mean of 47 months after SVR. ${ }^{5}$ It therefore remains uncertain if persistent virologic suppression to undetectable levels of HCV RNA impacts the risk of developing HCC in patients with severe hepatic fibrosis. Since cirrhosis is an independent risk factor for HCC, a vigilant monitoring of patients with HCV-related cirrhosis (compensated and decompensated) should be mandatory, even if SVR has been achieved after receiving interferon-based therapy.

\section{REFERENCES}

1. Cheinquer $\mathrm{N}$, Cheinquer $\mathrm{H}$, Wolff $\mathrm{FH}$ et al. Effect of sustained virologic response on the incidence of hepatocellular carcinoma in patients with HCV cirrhosis. Braz J Infect Dis 2010; 14(5):457-61.

2. Singal AK, Freeman DH Jr, Anand BS. Metaanalysis: interferon improves outcomes following ablation or resection of hepatocellular carcinoma. Aliment Pharmacol Ther 2010; 32:851-8.

3. Di Bisceglie AM, Shiffman ML, Everson GT et al. Prolonged therapy of advanced chronic hepatitis $\mathrm{C}$ with low-dose peginterferon. $\mathrm{N}$ Engl J Med 2008; 359:2429-41.

4. Trapero-Marugán M, Mendoza J, Chaparro M et al. Long-term outcome of chronic hepatitis C patients with sustained virological response to peginterferon plus ribavirin. World J Gastroenterol 2011; 17:493-8.

5. Ferreira Sda C, Carneiro Mde V, Souza FF et al. Long-term follow-up of patients with chronic hepatitis $C$ with sustained virologic response to interferon. Braz J Infect Dis 2010; 14:330-4. 